\title{
Influence of Different Bioactive Tooth Colored Restorative Materials in Caries Detection Using Cone Beam Computed Tomography
}

\author{
Ola. M. Sakr ${ }^{1,2, *}$, Iman Dakhli ${ }^{3}$ \\ ${ }^{1}$ Department of Conservative Dentistry, College of Dentistry, Qassim University, Kingdom of Saudi Arabia \\ ${ }^{2}$ Department of Operative Dentistry, College of Dentistry, Misr University for Science and Technology, Egypt \\ ${ }^{3}$ Department of Oral and Maxillofacial Radiology, Faculty of Dentistry, Cairo University, Egypt \\ *Corresponding author: olasakr2004@yahoo.com
}

\begin{abstract}
Aim: To ensure the diagnostic ability of CBCT for caries detection under different types of tooth colored restorative materials. Material and Methods: In the present study a total of 80 extracted carious permanent molar teeth were selected. Caries was classified as grade 3 according to International Caries Detection and Assessment System (ICDAS) criteria. Half of carious lesions were removed from 40 molars as Control specimens. Carious teeth were randomly assigned into two groups and each group was divided into four subgroups including: two carious and two non-carious as controlled group and all the teeth were filled with different bioactive restorative materials, then all the specimens were scanned with cone beam computed tomography (CBCT) using a Promax ${ }^{\circledR}$ 3DMid CBCT device (Planmeca, Oy, Helsinki, Finland). Results: Caries was detected in all the carious specimens of all the groups except that group A2 was much less remarkable than other groups. In the control specimens (noncarious) of all the groups: No caries was detected .Conclusion: Although CBCT is an excellent tool for secondary caries detection under different types of restorative materials but it should not be used as a primary diagnostic tool on regular basis. If its use is a must, so, optimized low dose low resolution protocol is recommended.
\end{abstract}

Keywords: caries detection, $C B C T$, bioactive restorative materials, recurrent caries

Cite This Article: Ola M, Sakr, and Dakhli I, "Influence of Different Bioactive Tooth Colored Restorative Materials in Caries Detection Using Cone Beam Computed Tomography." International Journal of Dental Sciences and Research, vol. 6, no. 4 (2018): 105-109. doi: 10.12691/ijdsr-6-4-6.

\section{Introduction}

Recurrent or secondary caries is a carious sore happening at the interproximal edge of a current restoration, particularly in territories of biofilm gathering, for example, the cervical edges. Recurrent caries is once in a while observed on occlusal surfaces of the teeth that those edges are effortlessly cleaned. Strikingly, around 50 to $60 \%$ of restorations are supplanted on account of a determination of recurrent caries [1-6].

A visual clinical examination ought to be joined with an exploring and radiographic examination, laser or light fluorescence-based techniques, electrical impedance estimations, ultrasound, MRI and so forth to enhance the identification of recurrent caries. It is generally acknowledged that radiography is the most widely recognized and valuable assistant for the finding of caries. The quick improvement of innovation has brought forth different advanced dental imaging modalities, including digital intraoral radiography and cone beam computerized tomography (CBCT) scanners. CBCT has made storming in maxillofacial imaging, encouraging the progress of dental determination from $2 \mathrm{D}$ to $3 \mathrm{D}$ pictures and extending the part of imaging from finding to imaging guidance of operative and surgical systems by utilization of outsider programming [2-11].

\section{Materials and Methods}

\subsection{Specimens Selection and Classification}

In the present study a total of 80 extracted carious permanent molar teeth were selected. Individual tooth surfaces were hand scaled to remove any remaining soft and hard tissues. All the teeth were stored in distilled water at room temperature. Caries was classified as grade 3 according to International Caries Detection and Assessment System (ICDAS) criteria. Half of carious lesions were removed from 40 molars as Control Samples. Carious teeth were randomly assigned into two groups

Group A: 40 teeth divided into 4 subgroups

Sub group A1: 10 carious teeth, carious cavities filled with Filtek Z350 nanofilled composite

Sub group A1 control: 10 caries free teeth, prepared cavities filled with Filtek Z350 nanofilled composite

Sub group A2: 10 carious teeth, carious cavities filled with glass ionomer base and Filtek Z350 nanofilled composite as final restoration 
Sub group A2 control: 10 caries free teeth, prepared cavities filled with Vitrebond light cure glass ionomer base and Filtek Z350 nanofilled composite as final restoration

Group B: 40 teeth divided into 4 subgroups

Sub group B1: 10 carious lesion teeth, carious cavities filled with ACTIVIA Bioactive Restoratives Sub group B1 Control: 10 caries free teeth, prepared cavities filled with ACTIVIA Bioactive Restoratives

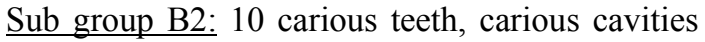
filled with ACTIVIA Bioactive base and ACTIVIA Bioactive Restoratives as final restoration

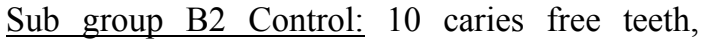
prepared cavities filled with ACTIVIA Bioactive base and ACTIVIA Bioactive Restoratives as final restoration. (Table 1).

\subsection{Preparation of the Specimens}

\subsubsection{Group A Specimens Preparation}

For subgroup A1 Cavities were etched for 10 seconds with $37 \%$ phosphoric acid, and rinsed with water spray for 10 seconds. Excess water was removed with cotton pellet or mini sponge. Bond (Adper Single bond 2) was applied with a disposable brush, 2 to 3 consecutive coats for 10 seconds with gentle agitation using a fully saturated applicator. Gently air thin for five seconds in evaporative solvents. Light cured for 10s using a halogen light source (Visulux curing unit, Vivadent; Schaan, Liechtenstein). The output of the light curing unit was regularly checked $\left(500 \mathrm{~mW} / \mathrm{mm}^{2}\right)$.

A nanofilled restorative composite (Filtek Z350 Shade A2, 3M, USA) was carefully applied into cavities and irradiated for 40 seconds according to the manufacturer's instructions. The specimens were then stored in artificial saliva at $37^{\circ} \mathrm{C}$ for $24 \mathrm{hrs}$.

For subgroup A2 cavities, firstly, were filled by Vitrebond light cure Glass ionomer base. One level scoop of loosely packed powder and one drop of liquid provide the recommended powder to liquid ratio. A small cement spatula used to rapidly mix (10-15 seconds) all the powder into the liquid. The mixed cement should have a smooth consistency and glossy appearance. The mixed liner/base applied to the dentin surfaces of the (carious or prepared) cavity in a thin layer $(1 / 2 \mathrm{~mm}$ or less) using a ball applicator. Vitrebond liner/base light cured for 30 seconds. Then, finally, Filtek Z350 Shade A2 was applied. With same steps mentioned in group A1

\subsubsection{Group B Specimens Preparation}

For subgroup B1, cavities were etched using 37\% phosphoric acid for 10 seconds, rinsed and dried, removing all excess moisture with a cotton pellet, A mix tip was placed on the ACTIVA syringe. Syringe was inserted into ACTIVA-SPENSER and snapped into place using firm pressure. Material was dispensed using gentle pressure. To ensure an even mix of base and catalyst, 1$2 \mathrm{~mm}$ of material was dispensed onto a mixing pad and discards this material.

ACTIVA was applied in increments up to $4 \mathrm{~mm} /$ increment, light curing for 20 seconds between each layer. Initial self-cure setting time is $2 \frac{1}{2}-3$ minutes. If allowing self-curing, exposed ACTIVA surfaces were covered with an oxygen barrier, e.g. glycerin. Self-curing capability is ideal for the bulk fill technique.

For subgroup B2 cavities were dried and removing excess moisture with cotton pellet. A mix tip was placed on the ACTIVA syringe. Syringe was inserted into ACTIVA-SPENSER and snapped into place using firm pressure. Material was dispensed using gentle pressure. To ensure an even mix of base and catalyst, $1-2 \mathrm{~mm}$ of material was dispensed onto a mixing pad and discards this material. ACTIVA Bioactive-BASE/LINER was applied to cavity dentin surfaces under final restoration then Light cured for 20 seconds. The restoration was completed along enamel surface with ACTIVA BioACTIVE-RESTORATIVE.

\subsection{Caries Detection of the Specimens}

All the specimens were embedded in mold of pink wax (Cavex Holland BV, Netherland) in the form of dental arch then scanned with CBCT using a Promax ${ }^{\circledR}$ 3DMid CBCT device (Planmeca,Oy, Helsinki, Finland) with following parameters (KvP 90, MA 8, exposure time $12.057 \mathrm{~s}$, voxel size $0.2 \mu)$.

Table 1. Composition, lot number, and manufacture of the tested materials

\begin{tabular}{|c|c|c|c|}
\hline Material & composition & Lot number & Manufacture \\
\hline Scotchbond etchant gel & $35 \%$ phosphoric acid & N 1201152 & 3M ESPE \\
\hline Adper Single bond 2 & $\begin{array}{l}\text { (10\% colloidal nanofiller) BisGMA, HEMA, dimethacrylates, ethanol, water, a novel } \\
\text { photoinitiator system and a methacrylate functional copolymer of polyacrylic and } \\
\text { polyitaconic acids }\end{array}$ & N43666 & 3M ESPE \\
\hline Filtek Z350 XT & $\begin{array}{l}\text { ( } 20 \mathrm{~nm} \text { silica filler } 4-11 \mathrm{~nm} \text { zirconia filler) as } 72.5 \% \text { by w filler bis-GMA, UDMA, } \\
\text { TEGDMA, PEGDMA and bis-EMA resins }\end{array}$ & N664814 & 3M ESPE \\
\hline $\begin{array}{l}\text { Vitrebond }{ }^{\mathrm{TM}} \text { Light Cure } \\
\text { Glass Ionomer } \\
\text { Liner/Base }\end{array}$ & $\begin{array}{l}\text { The powder component is a light sensitive fluoro-aluminosilicate glass. } \\
\text { The liquid component is a light sensitive polyalkenoic acid. }\end{array}$ & 7510 & 3M ESPE \\
\hline Bio active restoratives & $\begin{array}{l}56 \% \text { by weight reactive ionomer glass particles that mimic physical and chemical } \\
\text { properties of natural teeth., shock absorbing ionic resin component containing acidic } \\
\text { monomer with antimicrobial properties .no Bisphenol A, No BisGMA, no BPA } \\
\text { derivatives' }\end{array}$ & 150318 & $\begin{array}{l}\text { PULPDENT } \\
\text { Corporation }\end{array}$ \\
\hline Bio active base & $\begin{array}{l}45 \% \text { by weight bioactive fillers that mimic the physical and chemical properties of } \\
\text { natural teeth. It releases and recharges with calcium, phosphate and fluoride ions, } \\
\text { Strong, resilient ionic resin matrix. It chemically bonds to teeth, releases fluoride and } \\
\text { Contains no Bisphenol A, No Bis-GMA and no BPA derivatives. }\end{array}$ & 160408 & $\begin{array}{l}\text { PULPDENT } \\
\text { Corporation }\end{array}$ \\
\hline
\end{tabular}




\subsubsection{Visual Detection for Caries under Different Restorative Materials}

The CBCT caries detection was done using "Planmeca Romexis viewer 4.4.0.R". The adjustment of the image contrast and brightness by the operator to reach a subjective, personal optimum was done using built-in manipulative tools supplied by the machine software. (Figure 1). One independent well-trained radiologist with experience more than 10 years made all the CBCT caries detection after appropriate training and working on the software used in the current study (Planmeca Romexis viewer 3.5.1.R).
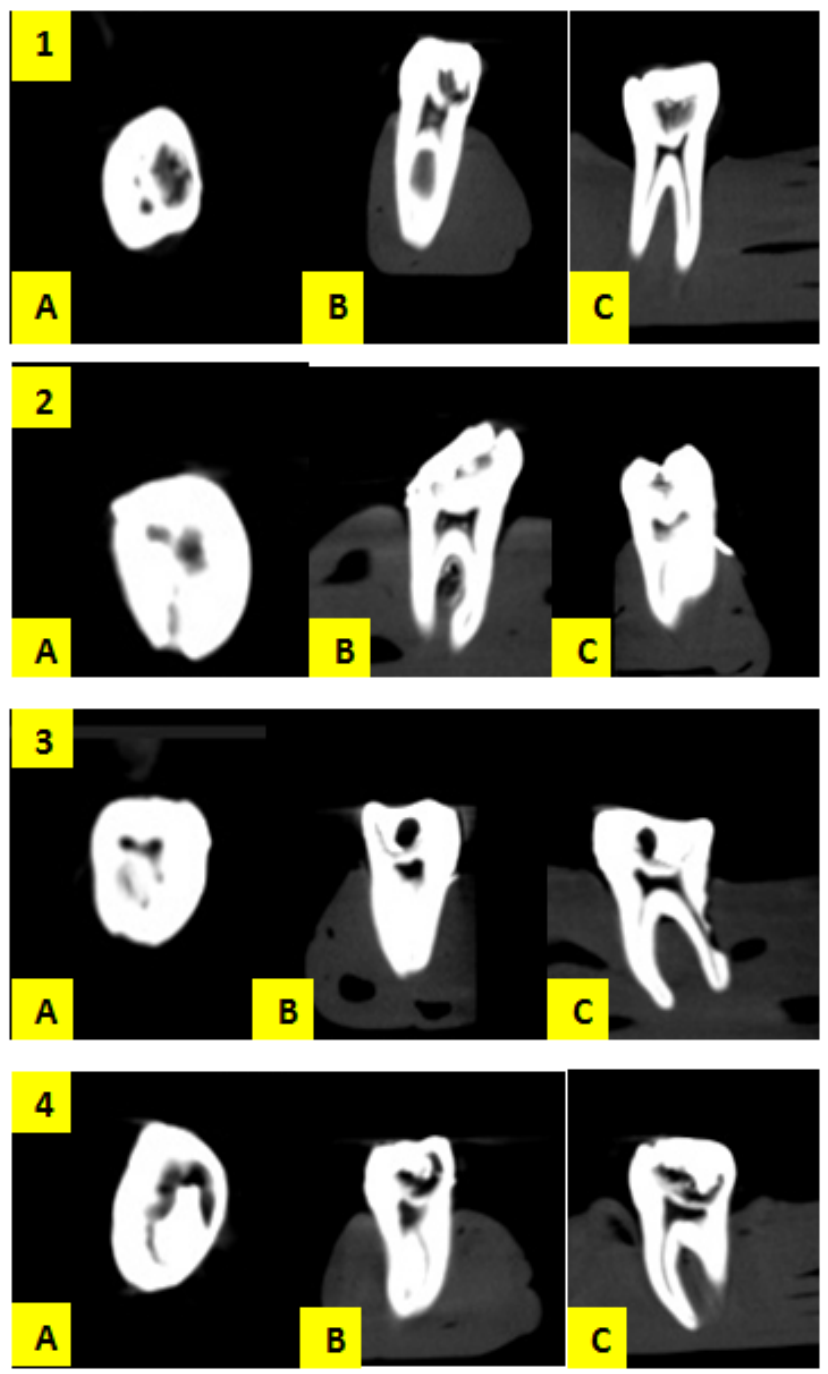

Figure 1. Showing four $\mathrm{CBCT}$ pictures represent the four groups conducted in the current study as follow: picture 1 represents group A1, picture 2 represents group A2, picture 3 represents group B1 and picture 4 represents group B2. In all pictures, A represents axial cut, B represents coronal cut and $\mathrm{C}$ represents sagittal cut. Caries was detected in all $\mathrm{CBCT}$ cuts

\section{Results}

Caries was detected in all the carious samples of all the groups except that group A2 was much less remarkable than other groups.

In the control samples (non-carious) of all the groups: No caries was detected.

\section{Discussion}

The present study verified the hypothesis stands for the superior diagnostic ability of CBCT for detection of secondary caries.

In spite of the advances in composite restorative materials and dentin bonding frameworks, secondary caries is yet a primary driver for failure of resin restorations. Precise, early detection of recurrent caries is the key for progress and life span of dental restorations. Radiography is among the most essential techniques for detection of caries, especially in the posterior teeth. The primary diagnosis of recurrent caries around composite restorations is especially vital in light of the fact that these restorations lack self-sealing and antibacterial properties. Resin restorations of the posterior teeth must be radiopaque because detection of marginal defects overhangs and secondary caries around these restorations exceptionally relies upon the radiopacity of these restorations [12].

The high diagnostic accuracy of CBCT frameworks is because of the way that they empower simple perception of all tooth surfaces in sagittal, coronal and axial planes and any gap or caries around the restorations are viewed as lucent zones at the tooth- restoration interface. In any case, in metal restorations, metal artifacts on the cavity walls compromise precise appraisal of the walls for caries which can dramatically reduce image quality and effectively produce caries-like 'lesions' in teeth. Therefore, the evidence indicates that CBCT should not be used for the purpose of caries diagnosis. Titanium and zirconia restorations as well as composite resins containing a minimum of $20 \% \mathrm{AlSiO} 2$ cause clear artifacts on CBCT scans. The intensity of artifacts increases by an increase in the radiopacity of composite resins. In the present study, the cavity walls were effortlessly evaluated because of the nonappearance of artifacts $[4,6,12,13,14]$.

The utilization of CBCT in routine clinical practice is impractical. However, CBCT examinations performed for different purposes it might be utilized as a subordinate for caries identification. Regardless of the conceivable favorable circumstances in analytic result of CBCT, it ought to be borne as a main priority that despite everything it brings about higher radiation dosages than conventional imaging options and intraoral examinations. Based on a systemic literature review, guidelines for the application of the CBCT have been detailed in the SEDENTEXCT project in Europe. These guidelines don't suggest CBCT for caries identification and analysis principally in light of the higher radiation dosage included contrasted with different types of intraoral radiography $[2,3,15,16,17,18]$.

Since the radiation dose is increased when taking a CBCT image with a high spatial resolution, so, low resolution low radiation dose protocol is recommended for evaluating dentinal caries when necessary as the spatial resolution did not have any impact on the detection accuracy of proximal caries. The CBCT images should not be used for evaluating carious lesions confined in enamel $[8,19,20]$.

In the current study, A2 carious group, caries detection was less remarkable than the other groups owing to type of the filling material as the use of glass ionomer base under Filtek Z350 nanofilled composite as final restoration has dissolved in the underlying dentine makes 
the caries detection less recognizable as the glass ionomer has chemical bond with dentine. This justification regarding the glass ionomer was in line with previous studies reports [23,24,25,26,27].

As almost the greater part of the investigations was done in vitro, So, their impediments were in accordance with our fundamental restrictions of the present investigation which is the in vitro think about recreated a perfect condition that avoids object movement, other teeth and tissue around the teeth, and other parameters that could create artifacts and convolute the diagnosis of caries. These might be viewed as the confinements of the present investigation.

Owing to $3 \mathrm{~d}$ capability of $\mathrm{CBCT}$, it has shown promising results in terms of the detection of caries lesions. CBCT system was evident superior to conventional and digital radiography for in vitro assessment of proximal caries lesion depth. Other factors that also compromise image quality of these systems include the voxel size, detector and FOV (field of view). As caries depth is also crucial for the diagnostic accuracy of radiographic systems. In respect to voxel size, Surprisingly, insignificant differences among machines with different voxel sizes $(0.076 \mathrm{~mm}$ to $0.322 \mathrm{~mm})$ was found on proximal caries diagnosis in previous study comparing five different $\mathrm{CBCT}$ machines regarding caries detection [1,10,20,28,29].

\section{Conclusion}

Although CBCT is an excellent tool for secondary caries detection under different types of restorative materials but it should not be used as a primary diagnostic tool on regular basis. If its use is a must, so, optimized low dose low resolution protocol is recommended.

\section{References}

[1] Qu X, Li G, Zhang Z, Ma X, " Detection accuracy of in vitro approximal caries by cone beam computed tomography images, " Eur J Radiol, 79(2). e24-7. 2011.

[2] Zhang ZL, Qu XM, Li G, Zhang ZY, Ma XC, "The detection accuracies for proximal caries by conebeam computerized tomography, film, and phosphor plates," Oral Surg Oral Med Oral Pathol Oral Radiol Endod, 111(1). 103-8. 2011.

[3] Baltacio`glu IH, Eren H, Yavuz Y, Kamburo־glu K, "Diagnostic accuracy of different display types in detection of recurrent caries under restorations by using CBCT," Dentomaxillofac Radiol, 2016. 45: 20160099.

[4] Vedpathak PR, Gondivkar SM, Bhoosreddy AR, Shah KR, Verma $\mathrm{GR}^{1}$, Mehrotra $\mathrm{GP}^{1}$, Nerkar $\mathrm{AC}^{1}$, "Cone Beam Computed Tomography:An Effective Tool in Detecting Caries Under Fixed Dental Prostheses" J Clin Diagn Res, 10(8). ZC10-3. 2016.

[5] Kamburoğlu K, Sönmez G, Berktaş ZS, Kurt H, Özen D, "Effects of various cone beam computed tomography settings on the detection of recurrent caries under restorations in extracted primary teeth", Imaging Sci Dent, 47(2).109-115. 2017.

[6] Sousa Melo SL, Furtado Belem MD, Prieto LT, Machado Tabchoury CP, Haiter-Neto F, "Comparison of cone beam CT and digital intraoral radiography performance in the detection of artificially induced recurrent caries-like lesions" Oral Surg Oral Med Oral Pathol Oral Radiol, 124(3). 306-314. 2017.

[7] Young SM, Lee JT, Hodges RJ, Chang TL, Elashoff DA, White $\mathrm{SC}$, "A comparative study of high-resolution cone beam computed tomography and charge-coupled device sensors for detecting caries" Dentomaxillofac Radiol, 38. 445-51. 2009.
[8] Cheng JG, Zhang ZL, Wang XY, Zhang ZY, Ma XC, Li G,"Detection accuracy of proximal caries by phosphor plate and conebeam computerizedtomography images scanned with different resolutions" Clin Oral Investig, 16(4). 1015-21. 2012.

[9] Kamburoglu K, Kurt H, Kolsuz E, O” ztas, B, Tatar I, Celik HH, "Occlusal caries depth measurements obtained by five different imaging modalities" J Digit Imaging, 24. 804-13. 2011

[10] Qu X, Li G, Zhang Z, Ma X, "Detection accuracy of in vitro approximal caries by cone beam computed tomography images" Eur J Radiol, 23, 2010 [Epub ahead of print].

[11] Murat S, Kamburo glu K, Isayev A, Kurs, un S, Y“uksel S, "Visibility of artificial buccal recurrent caries under restorations using different radiographic techniques" Oper Dent, 38. 197-207. 2013.

[12] Kasraei S, Shokri A, Poorolajal J, Khajeh S, Rahman $\mathrm{H}$,"Comparison of ConeBeamComputedTomography and Intraora Radiography in Detection ofRecurrent Caries under Composite Restorations" Braz Dent J, 28(1). 85-91. 2017.

[13] European Commission. Radiation Protection 172, "Evidence Based Guidelines on Cone Beam CT for Dental and Maxillofacial Radiology. Luxembourg" Office for Official Publications of the European Communities: 2012. Available from: http://ec.europa.eu/energy/nuclear/radiation.protection/doc/publica tion/172.pdf.

[14] Horner K, "Cone-beam computed tomography: time for an evidence-based approach" Prim Dent J, 2(1). 22-31. 2013.

[15] Ludlow JB, Davies-Ludlow LE, Brooks SL, Howerton WB, "Dosimetry of $3 \mathrm{CBCT}$ devices for oral and maxillofacial radiology: CB Mercuray, NewTom $3 \mathrm{G}$ and i-CAT" Dentomaxillofac Radiol, 35.219-226. 2006.

[16] Ludlow JB, Davies-Ludlow LE, White SC, "Patient risk related to common dental radiographic examinations: the impact of 2007 International Commission on Radiological Protection recommendations regarding dose calculation" J Am Dent Assoc, 139. 1237-1243. 2008

[17] Rathore S, Tyndall D, Wright J, Everett E, "Ex vivo comparison of Galileos cone beam CT and intraoral radiographs in detecting occlusal caries" Dentomaxillofac Radiol, 41. 489-93. 2012.

[18] Cebe F, Aktan AM, Ozsevik AS, Ciftci ME, Surmelioglu HD, "The Effect of Different Restorative Materials on the Detection of Approximal Caries in Cone Beam Computed Tomography Scans with and without the Metal Artefact Reduction Mode" Oral Surg Oral Med Oral Pathol Oral Radiol, 123(3). 392-400. 2017.

[19] Haiter-Neto F, Wenzel A, Gotfredsen E, "Diagnostic accuracy of cone beam computed tomography scans compared with intraoral image modalities for detection of caries lesions" Dentomaxillofac Radiol, 37. 18-22. 2008

[20] Kamburo glu K, Murat S, Yuksel SP, Cebeci AR, Paksoy CS, "Occlusal caries detection by using a cone-beam CT with different voxel resolutions and a digital intraoral sensor" Oral Surg Oral Med Oral Pathol Oral Radiol Endod, 109(5). 63-9. 2010.

[21] Qu XM, Li G, Ludlow JB, Zhang ZY, Ma XC, "Effective radiation dose of ProMax 3D cone-beam computerized tomography scanner with different protocols" Oral Surg Oral Med Oral Pathol Oral Radiol and Endod, 110. 770-776. 2010.

[22] Pauwels R, Beinsberger J, Collaert B et al, "Effective dose range for dental cone beam computed tomography scanners" Eur $J$ Radiol, 2010.

[23] Hewlett ER, Caputo AA, Wrobet DC, "Glass ionomer bond strength and treatment of dentin with polyacrylic acid" J Prosthet Dent, 66(6). 767-72. 1991

[24] Berry EA, Powers JM, "Bond strength of glass ionomers to coronal and radicular dentin" Oper Dent, 19. 122-6.1994.

[25] Ewoldsen N, Covey D, Lavin M, "The physical and adhesive properties of dental cements used for atraumatic restorative treatment" Spec Care Dentist, 17 (1). 9-24.1997.

[26] Pereira PN, Yamada T, Inokoshi S, Burrow MF, Sano H, Tagami J "Adhesion of resin-modified glass-ionomer cement using resinbonding systems " J Dent, 26.479-85.1998.

[27] Yap AU, Tan AC, Goh AT, Goh DC, Chin KC, "Effect of surface treatment and cement maturation on the bond strength of resin modified glass ionomers to dentin", Oper Dent, 28(6). 728-33, 2003.

[28] Kayipmaz S, Sezgin O* S, Saricaog lu ST, Can G," An in vitro comparison of diagnostic abilities of conventional radiography, 
storage phosphor, and cone beam computed tomography to determine occlusal and approximal caries" Eur J Radiol, 80. 478-82. 2011.
[29] Spin-Neto R, Gotfredsen E, Wenzel A, "Impact of voxel size variation on CBCTbased diagnostic outcome in dentistry: a systematic review" J Digit Imaging, 26(4). 813-20. 2013. 